\title{
Modal Parameter Identification Based on Hilbert Vibration Decomposition in Vibration Stability of Bridge Structures
}

\author{
Yingzhi Xia $\mathbb{D}^{1},{ }^{1}$ Hui Li $\mathbb{D},{ }^{1}$ Zhezhe Fan $\mathbb{D}^{1},{ }^{1}$ and Jiyong Xiao $\mathbb{D}^{2}$ \\ ${ }^{1}$ School of Civil and Transportation Engineering, Henan University of Urban Construction, Longxiang Road Xincheng Area, \\ Pingdingshan, Henan 467036, China \\ ${ }^{2}$ China Railway No. 10 Engineering Group Investment and Development Co., Ltd., Jinan, Shandong, 250000, China
}

Correspondence should be addressed to Yingzhi Xia; 30070501@hncj.edu.cn

Received 17 December 2020; Revised 17 January 2021; Accepted 30 January 2021; Published 26 February 2021

Academic Editor: Junhong Yuan

Copyright (C) 2021 Yingzhi Xia et al. This is an open access article distributed under the Creative Commons Attribution License, which permits unrestricted use, distribution, and reproduction in any medium, provided the original work is properly cited.

\begin{abstract}
Modal parameters are important parameters for the dynamic response analysis of structures. An output-only modal parameter identification technique based on Hilbert Vibration Decomposition (HVD) is developed herein for structural modal parameter identification to (1) obtain the Free Decay Response (FDR) of a structure through free vibration or ambient vibration tests, (2) decompose the FDR into modal responses using HVD, and (3) calculate the instantaneous frequencies and instantaneous damping ratios of the modal responses to obtain the modal frequencies and modal damping ratios. A series of numerical examples are examined to demonstrate the efficiency and highlight the superiorities of the proposed method relative to the empirical model decomposition-based (EMD-based) method. The robustness of the proposed method to noises is also investigated and proved to be positive effect. The proposed method is proved to be efficient in modal parameter identification for both linear and nonlinear systems, with better frequency resolution, and it can be applied to systems with closely spaced modes and low-energy mode.
\end{abstract}

\section{Introduction}

Modal parameters (including modal frequency and modal damping ratio) are important parameters for the dynamic response analysis of structures. Accurate and convenient identification of modal parameters is one of the research hotspots in the engineering field. Modal parameters can be identified by forced vibration, free vibration, or random vibration tests under environmental excitation. For large civil structures, the cost of forced vibration testing is high, and the continuous external excitation load may considerably damage the structure. Therefore, parameter identification methods based on free vibration or random vibration tests have attracted increasing attention. Because this kind of method does not need to measure the input, they are often called output-only modal analysis (OMA) methods [1].

Early OMA methods include the peak pick-up, frequency domain decomposition, random decrement, and random subspace methods. These methods are easy to implement, but their abilities are limited when dealing with structures with large damping, dense modes, or low-energy modes. In addition, the traditional methods cannot be used to analyze the nonlinear and nonstationary characteristics of structural dynamic responses.

Many scholars have proposed and developed modal parameter identification methods based on time-frequency analysis [2], including the more classical methods based on wavelet transform, empirical model decomposition (EMD), and Hilbert transform [3,4]. The analysis results of the wavelet transform method are sensitive to the selection of the mother wavelet function, and no general theory is available for choosing the mother wavelet function. The EMD method is a kind of adaptive signal decomposition technology that can be combined with Hilbert transform to form a powerful time-frequency analysis tool, usually called Hilbert Huang transform (HHT). Many scholars have studied modal parameter identification based on HHT method, introducing some improved algorithms to improve the performance of the HHT method [5]. 
Although modal parameter identification based on EMD has been widely used, it has some obvious defects: it cannot effectively decompose dense and low-energy modes, and it may produce pseudomodes. Hilbert vibration decomposition (HVD) [6, 7] is a more recently proposed adaptive signal decomposition method. It overcomes some defects of the EMD method and has been applied in vibration signal analysis [8], structural fault diagnosis, and modal parameter identification of linear systems [9]. Applications of HVD to the identification of modal parameters of nonlinear systems and its advantages over EMD are worthy of further study [10].

In this study, a new modal parameter identification method is proposed based on the HVD method. Through a series of numerical examples, the effectiveness and superiority of this method are verified.

\section{Hilbert Vibration Decomposition (HVD) Process}

In modal parameter identification, the multifrequency component signal (measured vibration response or free attenuation vibration response) must often be decomposed into a single-frequency component signal (modal response). Traditional signal decomposition usually adopts the EMD method and some improved EMD algorithms [4, 5]. The HVD method introduced in this study is an adaptive signal decomposition method recently proposed. Any continuous multifrequency component signal can be expressed as

$$
s(t)=\sum_{k=1}^{K} v_{k}(t)=\sum_{k=1}^{K} A_{k}(t) \cos \left[\int \omega_{k}(t) \mathrm{d} t+\theta_{k}\right],
$$

where $s(t)$ is the multifrequency component signal to be decomposed, $v_{k}(t)(k=1, \ldots, K)$ is the $k$ th-order component of $s(t), K$ is the number of decomposition layers, $A_{k}(t)$ and $\omega_{k}(t)$ are the instantaneous amplitude and the instantaneous circular frequency of $v_{k}(t)$, respectively, and $\theta_{k}$ is the initial phase angle of $v_{k}(t) . f_{k}(t)=\left(\omega_{k}(t) /(2 \pi) v_{k}(t)\right)$ is the instantaneous frequency.

2.1. Instantaneous Frequency Estimation of Single Frequency Component Signal. First, the analytical signal of $s(t)$ was obtained by carrying out Hilbert transformation consider the case of $K=2$ :

$$
\begin{aligned}
z(t)= & A_{1}(t) e^{j\left(\int \omega_{1}(t) \mathrm{d} t+\theta_{1}\right)} \\
& +A_{2}(t) e^{j\left(\int \omega_{2}(t) \mathrm{d} t+\theta_{2}\right)} .
\end{aligned}
$$

Assuming $A_{1}(t)>A_{2}(t)$, the instantaneous amplitude and the instantaneous circular frequency of $z(t)$ can be expressed as

$$
\begin{aligned}
& A(t)=\left[A_{1}^{2}(t)+A_{2}^{2}(t)+2 A_{1}(t) A_{2}(t) \cos \left(\int\left(\omega_{2}(t)-\omega_{1}(t)\right) \mathrm{d} t+\left(\theta_{2}-\theta_{1}\right)\right)\right]^{1 / 2}, \\
& \omega(t)=\omega_{1}(t)+\frac{\left[\omega_{2}(t)-\omega_{1}(t)\right]\left[A_{2}^{2}(t)+2 A_{1}(t) A_{2}(t) \cos \left(\int\left(\omega_{2}(t)-\omega_{1}(t)\right) \mathrm{d} t+\left(\theta_{2}-\theta_{1}\right)\right)\right]}{A^{2}(t)} .
\end{aligned}
$$

According to formula (4), $\omega(t)$ is equivalent to superimposing the high-frequency oscillation component on the instantaneous circular frequency $v_{1}(t)$ of the maximum amplitude component $\omega_{1}(t)$. Therefore, a low-pass filter can be applied to $\omega(t)$ to obtain $\omega_{1}(t)$. For $N>2, \omega(t)$ expression is more complex, but $\omega(t)$ can still be filtered to obtain the instantaneous circular frequency of the maximum amplitude component.

\subsection{Instantaneous Amplitude Estimation of Single Frequency} Component Signal. HVD is used to calculate the instantaneous amplitude of the maximum amplitude component by synchronous detection of amplitude and phase of $s(t)$. Generally, the instantaneous circle frequency obtained in Section 2.1 is recorded as $\omega_{r}(t)$. The product of $s(t)$ and the two orthogonal signals of $\cos \left[\int \omega_{r}(t) \mathrm{d} t\right]$ and $\sin \left[\int \omega_{r}(t) \mathrm{d} t\right]$ was shown as follows:

$$
s_{k=r}(t)=0.5 A_{k}(t)\left[\cos \left(\theta_{k}\right)+\cos \left(\int \omega_{k}(t)+\omega_{r}(t)+\theta_{k}\right)\right],
$$

$$
\widetilde{s}_{k=r}(t)=0.5 A_{k}(t)\left[\sin \left(\theta_{k}\right)+\sin \left(\int \omega_{k}(t)+\omega_{r}(t)+\theta_{k}\right)\right] .
$$

Conduct low-pass filtering for formulas (5) and (6):

$$
\begin{gathered}
s_{k=r}(t)=0.5 A_{k}(t) \cos \left(\theta_{k}\right), \\
\widetilde{s}_{k=r}(t)=0.5 A_{k}(t) \sin \left(\theta_{k}\right) .
\end{gathered}
$$

The instantaneous amplitude of the maximum amplitude component is

$$
A_{k=r}(t)=2 \sqrt{\left[\widetilde{s}_{k=r}(t)\right]^{2}+\left[\widetilde{s}_{k=r}(t)\right]^{2}} .
$$


2.3. Iterative Extraction of Single Frequency Component Signal. The maximum amplitude component obtained by the methods in Sections 2.1 and 2.2 is

$$
v_{r}(t)=A_{r}(t) \cos \left[\int \omega_{r}(t) \mathrm{d} t+\theta_{r}\right] .
$$

Subtract $v_{r}(t)$ from the decomposed signal to obtain the signal to be decomposed in the next iteration:

$$
s_{r}(t)=s_{r-1}(t)-v_{r}(t)
$$

where $r \geq 1$ and $s_{r}(t)$ is the residual after the second iteration, which is the signal to be decomposed in the $(r+1)$ th iterative step. The standard deviation of $s_{r}(t)$ can be used as the termination condition of the iteration. For example, when $\sigma<0.001$, the iteration is terminated.

\section{Method}

The modal parameter identification method proposed in this study can be divided into three steps: (1) obtain the free attenuated vibration response (FDR) of the structure from the random vibration signal of the structure using a free vibration test or the random decrement method, (2) extract the modal response from the FDR using the HVD method, and (3) calculate the instantaneous frequency and damping of each modal response and then obtain the modal frequency and damping. In the above steps, the structural vibration test was outside the scope of this study. The second step was discussed in detail in Section 2. This section introduces the content for step 3.

Assuming that the modal response of the structure was extracted using HVD, the instantaneous frequency and amplitude of $v i(t)$ can be calculated. Hilbert transform is the most commonly used method to solve the instantaneous frequency and amplitude of a signal. However, due to the limitation of the Bedrosian theorem and Nuttall theorem [11], the instantaneous frequency obtained by Hilbert transform may have obvious fluctuations. In addition, Hilbert transform produces an obvious end effect. To overcome these shortcomings of Hilbert transform, some scholars constructed improved algorithms. Among them, the results of the empirical envelope (EE) method are stable, the end effect is weak, and its advantages have been verified in the literature. Therefore, the EE method was used to solve the instantaneous frequency of modal response $[12,13]$.

For the time-varying damping signal, the signal damping can be solved by sections under the assumption that the signal damping remains constant for several periods. However, the instantaneous characteristics of the signal damping cannot be obtained using this method. In this study, the method in the literature is used to calculate the instantaneous damping of a single-frequency component signal [14].

According to $A_{k}(t)$, the instantaneous logarithmic decay rate of $v_{k}(t)$ can be calculated as

$$
\delta_{k}\left(t_{m}\right)=\ln \frac{A_{k}\left(t_{m}\right)}{A_{k}\left(t_{m-1}\right)}=\frac{2 \pi \xi_{k}\left(t_{m}\right)}{\sqrt{1-\xi_{k}^{2}\left(t_{m}\right)}},
$$

where $m$ represents the number of sampling points and $\xi_{k}(t)$ is the instantaneous damping ratio of $v_{k}(t)$, and

$$
\xi_{k}(t)=\frac{\ln \left[A_{k}\left(t_{m}\right) / A_{k}\left(t_{m-1}\right)\right]}{\sqrt{4 \pi^{2}+\left\{\ln \left[A_{k}\left(t_{m}\right) / A_{k}\left(t_{m-1}\right)\right]\right\}^{2}}} .
$$

For the case of small damping, $\ln \left[q\left(t_{m}\right) / q\left(t_{m-1}\right)\right]<<2 \pi$, formula (12) can be simplified as

$$
\xi_{k}(t)=\frac{\ln \left[A_{k}\left(t_{m}\right) / A_{k}\left(t_{m-1}\right)\right]}{2 \pi} .
$$

Formula (10) does not involve differential operation, is less affected by noise, and is not affected by instantaneous frequency results, so the calculation results are relatively stable. Notably, the instantaneous damping ratio obtained by formula (10) may fluctuate due to the interference of discrete sampling and noise. In practical applications, the instantaneous damping ratio of the signal can be averaged over a certain period of time (such as a single vibration period) to increase the smoothness of the result.

For linear systems, the instantaneous modal frequency and damping ratio are approximately constant, while they will be changed with time for nonlinear systems. Experimental results showed that the modal frequency and modal damping ratio of a large number of civil structures often change with the amplitude $[2,5,14]$. Therefore, the instantaneous modal frequency and modal damping ratio can be transformed into the modal frequency and modal damping ratio with amplitude change using least square fitting:

$$
\begin{gathered}
{\left[A_{k}(t), f_{k}(t)\right] \stackrel{\text { Fitting }}{\longrightarrow} f_{k}\left(A_{k}\right),} \\
{\left[A_{k}(t), \xi_{k}(t)\right] \stackrel{\text { Fitting }}{\longrightarrow} \xi_{k}\left(A_{k}\right) .}
\end{gathered}
$$

For linear systems, $f_{k}\left(A_{k}\right)$ and $\xi_{k}\left(A_{k}\right)$ are approximately constant. For nonlinear systems, the variations of $f_{k}\left(A_{k}\right)$ and $\xi_{k}\left(A_{k}\right)$ reflect the variation trend of the structural modal frequency and the modal damping ratio with amplitude.

\section{Results}

The HVD method overcomes some shortcomings of the EMD method, maintains the ability to decompose multifrequency component signals, and detects the amplitude or frequency transient changes of signals. Therefore, HVD performs better than EMD in the decomposition or time-frequency analysis of specific types of signals and the modal parameter identification of certain types of structures. In this section, three numerical examples are used to verify the effectiveness and superiority of the modal parameter identification method. The head and tail results were excluded from the example results due to the certain end effect of the decomposition results of the HVD method. The end effect of HVD can be weakened by means of signal extension.

4.1. Two Frequency Component Signals with Closely Spaced Modes. Considering a two degree-of-freedom linear system with dense modes, its FDR is shown in formula (16), where $\xi_{1}=\xi_{2}=0.01, f_{1}=3.5 \mathrm{~Hz}$, and $f_{2}=4 \mathrm{~Hz}$. The time history and amplitude spectrum of the FDR (sampling frequency $50 \mathrm{~Hz}$ ) shown in formula (16) are depicted in Figure 1: 

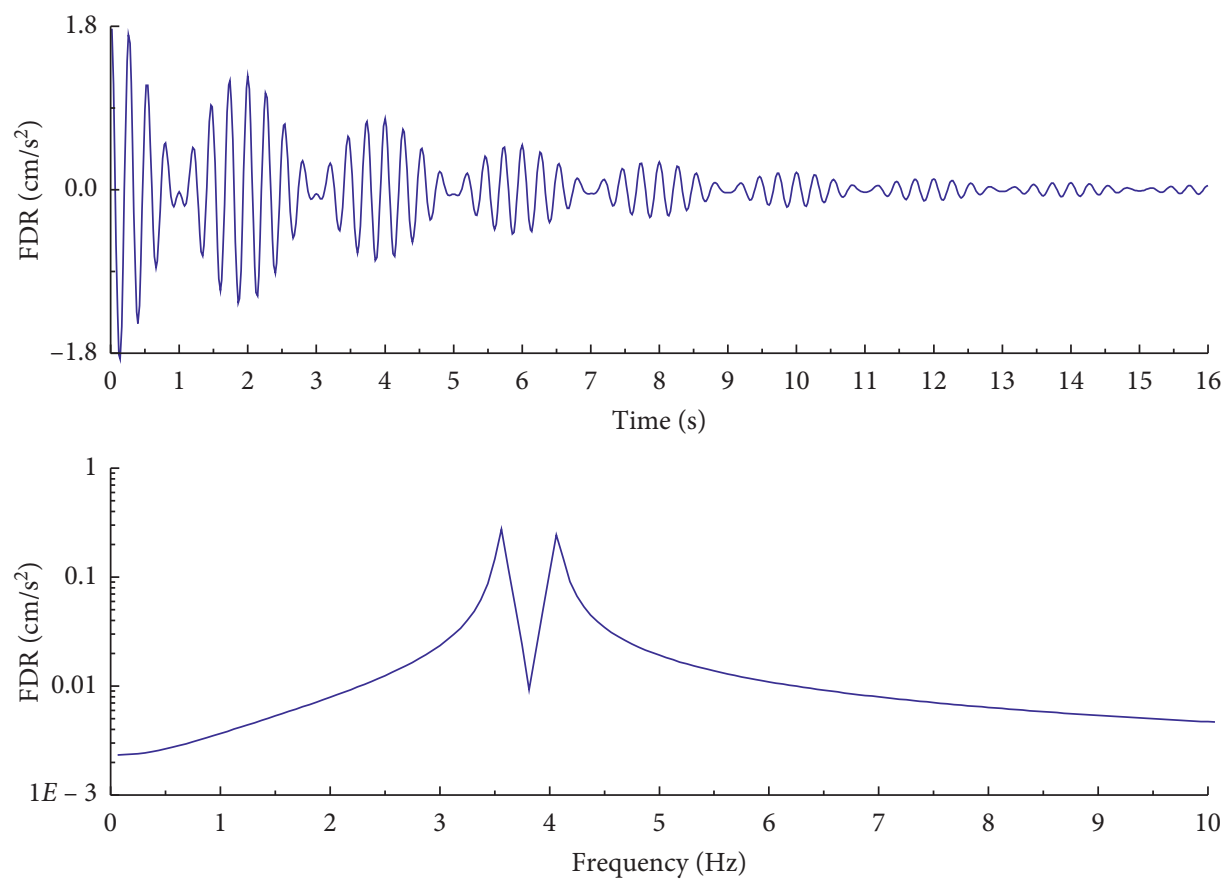

Figure 1: Two-component signal with closely spaced modes.

$$
\begin{aligned}
s(t)= & v_{1}(t)+v_{2}(t)=e^{-2 \pi \xi_{1} f_{1} t} \cos \left(2 \pi f_{1} t\right) \\
& +e^{-2 \pi \xi_{2} f_{2} t} \cos \left(2 \pi f_{2} t\right) .
\end{aligned}
$$

The FDR is decomposed into two modal responses using the HVD method, and the decomposition result and the real modal response are compared in Figure 2. The HVD method can accurately decompose two modal responses in FDR. The EMD method was used to decompose the FDR to verify the advantages of the HVD method, as shown in Figure 1, and the results are shown in Figure 3. Figure 3 shows that the EMD decomposition results overlap due to the close frequency of the two modes, which leading to the two-mode response in FDR cannot be decomposed. In addition, there are many pseudomodes in the EMD decomposition results, which may mislead users when dealing with practical projects. These results indicated that the HVD method has better frequency resolution than the EMD method.

The instantaneous amplitudes of $v_{1}(t)$ and $v_{2}(t)$ are shown in Figure 2. The instantaneous amplitude results agree well with the signal peak value, reflecting the recognition accuracy of the instantaneous amplitude. The identification results of the instantaneous frequency and instantaneous damping ratio of $v_{1}(t)$ and $v_{2}(t)$ are shown in Figure 4 , which indicates a well agreement with the theoretical values. The results show that this method is suitable for the identification of modal parameters of structures with closely spaced modes.

To investigate the identification accuracy of different damping ratio systems using this method, the damping ratio in formula (16) was changed to $\xi_{1}=\xi_{2}=0.02,0.04,0.06$, and 0.08. Table 1 lists the identification results of the modal parameters under different damping ratios. When the damping ratio is as high as 0.06 , the accuracy of the proposed method is still satisfactory; when the damping ratio continues to increase, the identification results of the modal frequency and modal damping ratio show some errors. Due to the high damping ratio, the signal attenuation is fast and the available signal is short, so the HVD method struggles to accurately decompose two adjacent modes.

To investigate the influence of noise on the identification accuracy of this method, zero mean Gaussian white noise was added to the FDR shown in Figure 1. Table 2 lists the identification results of modal frequency and modal damping ratio under different signal-to-noise ratios (SNR). Therefore, this method has good antinoise performance.

4.2. Two Frequency Component Signals with Low-Energy Mode. The FDR of a two-degrees-of-freedom linear system is shown in formula (13), where $\xi_{1}=\xi_{2}=0.01, f_{1}=2 \mathrm{~Hz}$, and $f_{2}=4 \mathrm{~Hz}$, and the time history and amplitude spectrum of the FDR (sampling frequency $50 \mathrm{~Hz}$ ) are shown in Figure 5. Formula (17) shows that the $v_{2}(t)$ energy is only about $10 \%$ of the $v_{1}(t)$ energy in FDR:

$$
\begin{aligned}
s(t)= & v_{1}(t)+v_{2}(t)=e^{-2 \pi \xi_{1} f_{1} t} \cos \left(2 \pi f_{1} t\right) \\
& +0.1 e^{-2 \pi \xi_{2} f_{2} t} \cos \left(2 \pi f_{2} t\right) .
\end{aligned}
$$

The FDR is decomposed into two modal responses using the HVD method, and the decomposition results and the real modal response are compared in Figure 6. The HVD method can accurately decompose two modal responses in FDR. The EMD method was used to decompose the FDR to verify the advantages of the HVD method as shown in Figure 5, and the results are shown in Figure 7. Figure 7 shows that EMD cannot decompose the two modal response due to the low $v_{2}(t)$ energy 

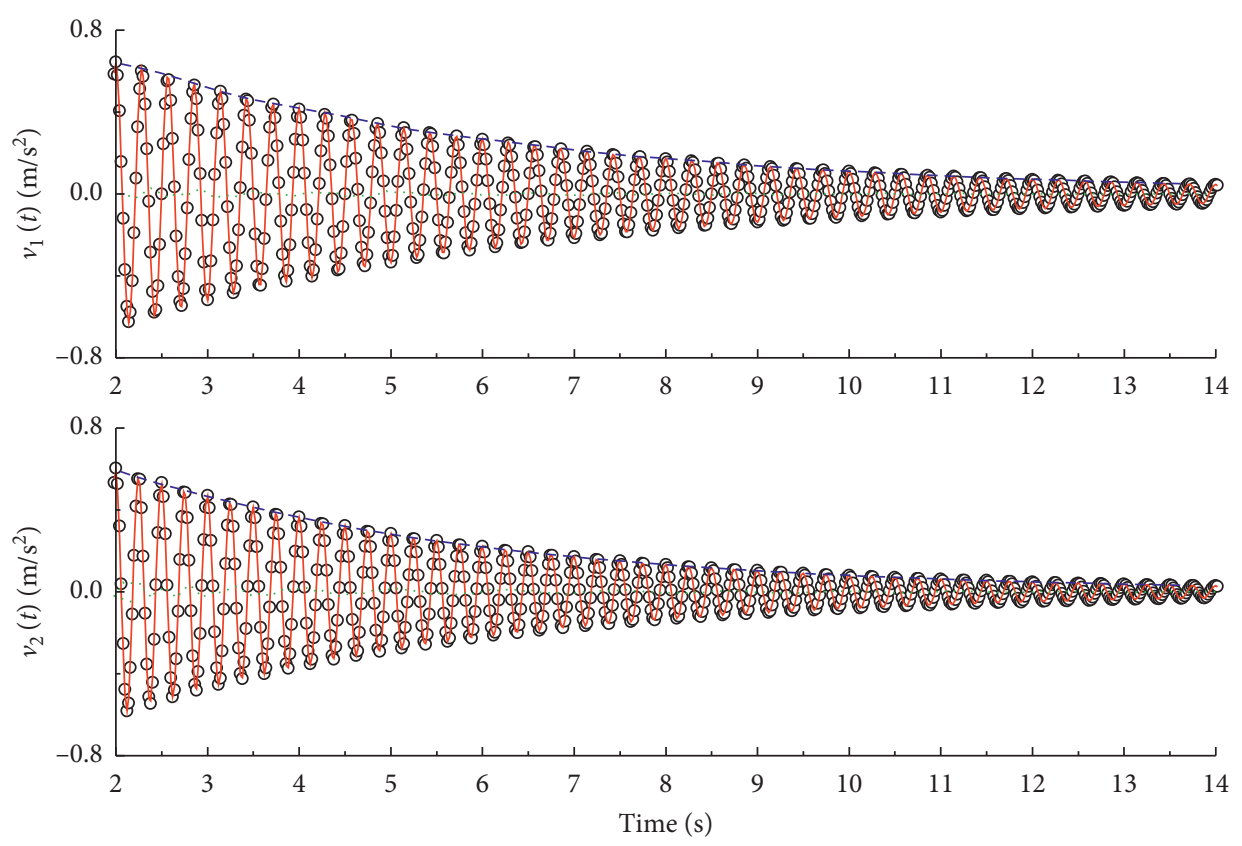

- True value

Error

— HVD

-- - Synchronic amplitude

Figure 2: Modal responses of 2 degree-of-dreedom (DOF) system (Hilbert vibration decomposition (HVD) results). $v_{1}$ and $v_{2}$ are the maximum amplitude components under the instantaneous frequency of $\mathrm{f}_{1}=3.5 \mathrm{~Hz}$ and $\mathrm{f}_{2}=4 \mathrm{~Hz}$.
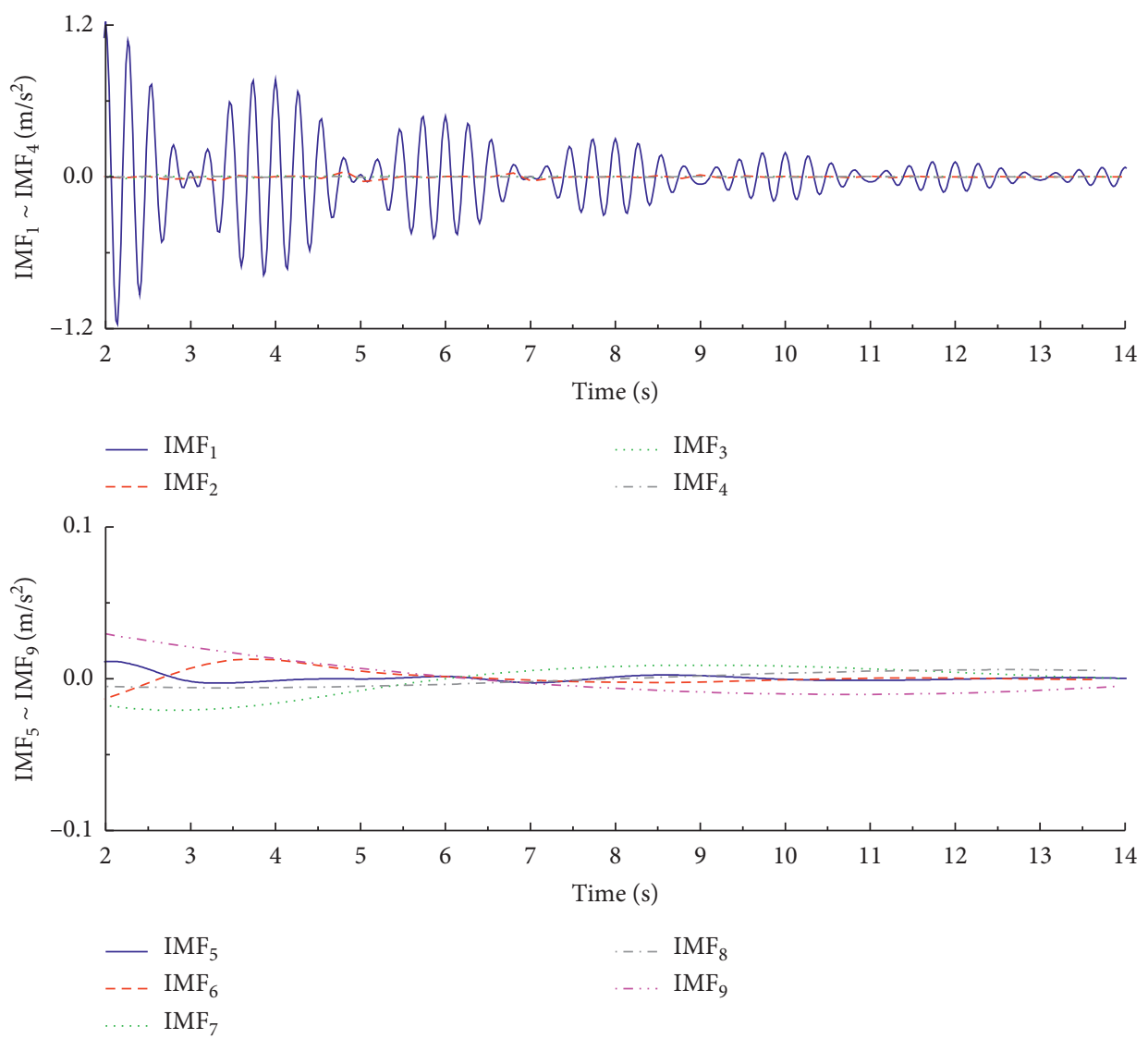

Figure 3: Empirical model decomposition results with closely spaced modes. 

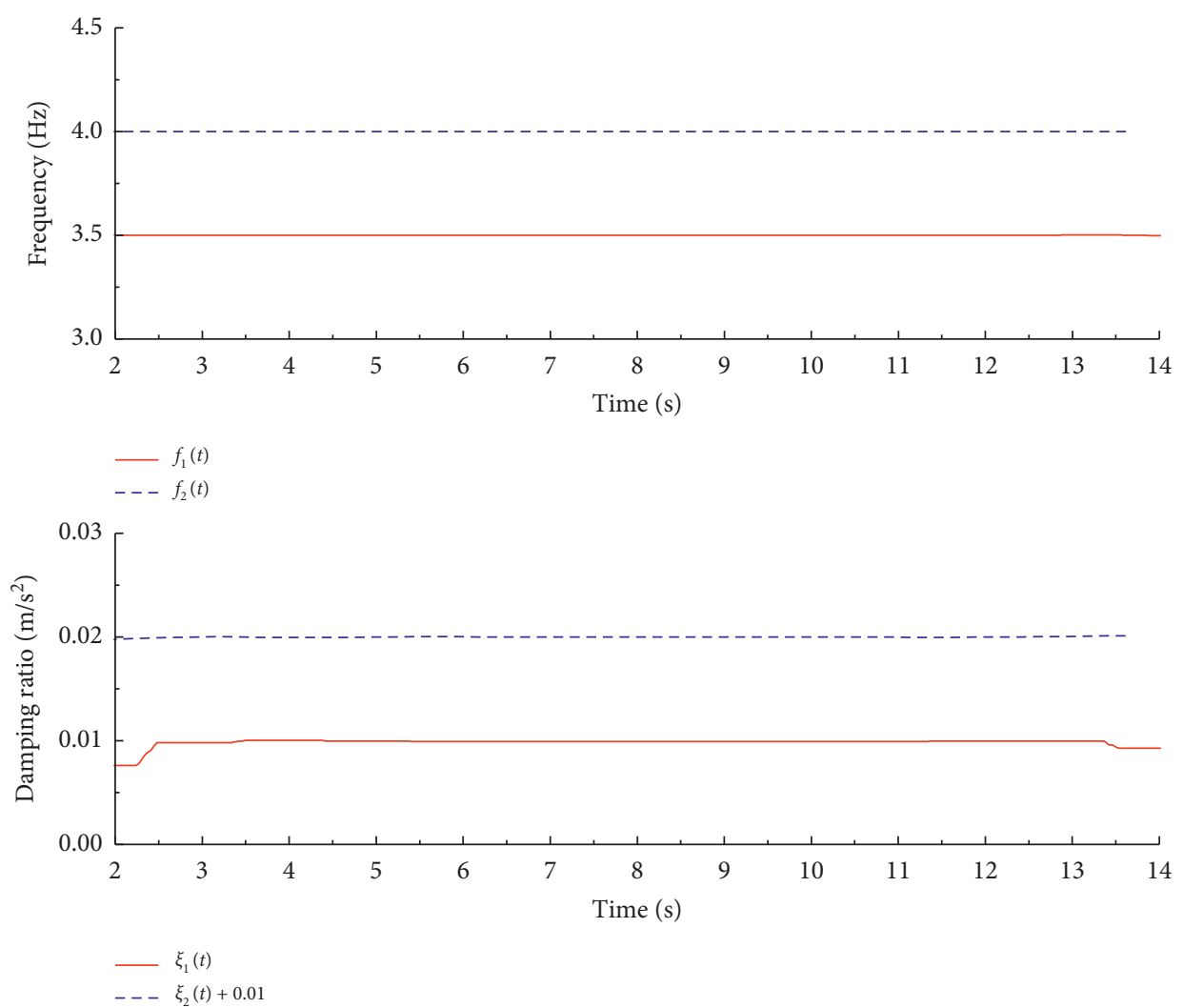

Figure 4: Instantaneous frequency and damping ratio.

TABLE 1: Identification results for various damping ratios.

\begin{tabular}{lcccc}
\hline$\xi_{1}=\xi_{2}$ & $f_{1}$ & $f_{2}$ & $\xi_{1}$ & $\xi_{2}$ \\
\hline 0.01 & 3.50 & 4.00 & 0.010 & 0.010 \\
0.02 & 3.50 & 4.00 & 0.020 & 0.020 \\
0.04 & 3.50 & 4.00 & 0.010 & 0.010 \\
0.06 & 3.50 & 3.91 & 0.061 & 0.063 \\
0.08 & 3.56 & 3.76 & 0.082 & 0.074 \\
\hline
\end{tabular}

Note. $\xi_{1}$ and $\xi_{2}$ are the modal damping and $f_{1}$ and $f_{2}$ are the modal frequency.

TABLE 2: Identification results for various signal-to-noise ratios (SNRs) with closely spaced modes.

\begin{tabular}{lcccc}
\hline SNR $(\mathrm{db})$ & $f_{1}$ & $f_{2}$ & $\xi_{1}$ & $\xi_{2}$ \\
\hline$+\infty$ & 3.50 & 4.00 & 0.010 & 0.010 \\
40 & 3.50 & 4.00 & 0.010 & 0.010 \\
30 & 3.50 & 4.00 & 0.010 & 0.010 \\
20 & 3.50 & 4.00 & 0.010 & 0.010 \\
10 & 3.50 & 4.00 & 0.010 & 0.010 \\
\hline
\end{tabular}

Note. $\xi_{1}$ and $\xi_{2}$ are the modal damping and $f_{1}$ and $f_{2}$ are the modal frequency.

ratio in FDR. The HVD method can separate low-energy modes better than the EMD method.

The identification results of the instantaneous frequency and instantaneous damping ratio of $v_{1}(t)$ and $v_{2}(t)$ are shown in Figure 8, which indicate a well agreement with the theoretical values, thus verifying the accuracy of this method. The results showed that this method can be used to identify the low-energy mode modal parameters.

The results of modal frequency and modal damping ratio identification under different SNR are provided in Table 3. The 

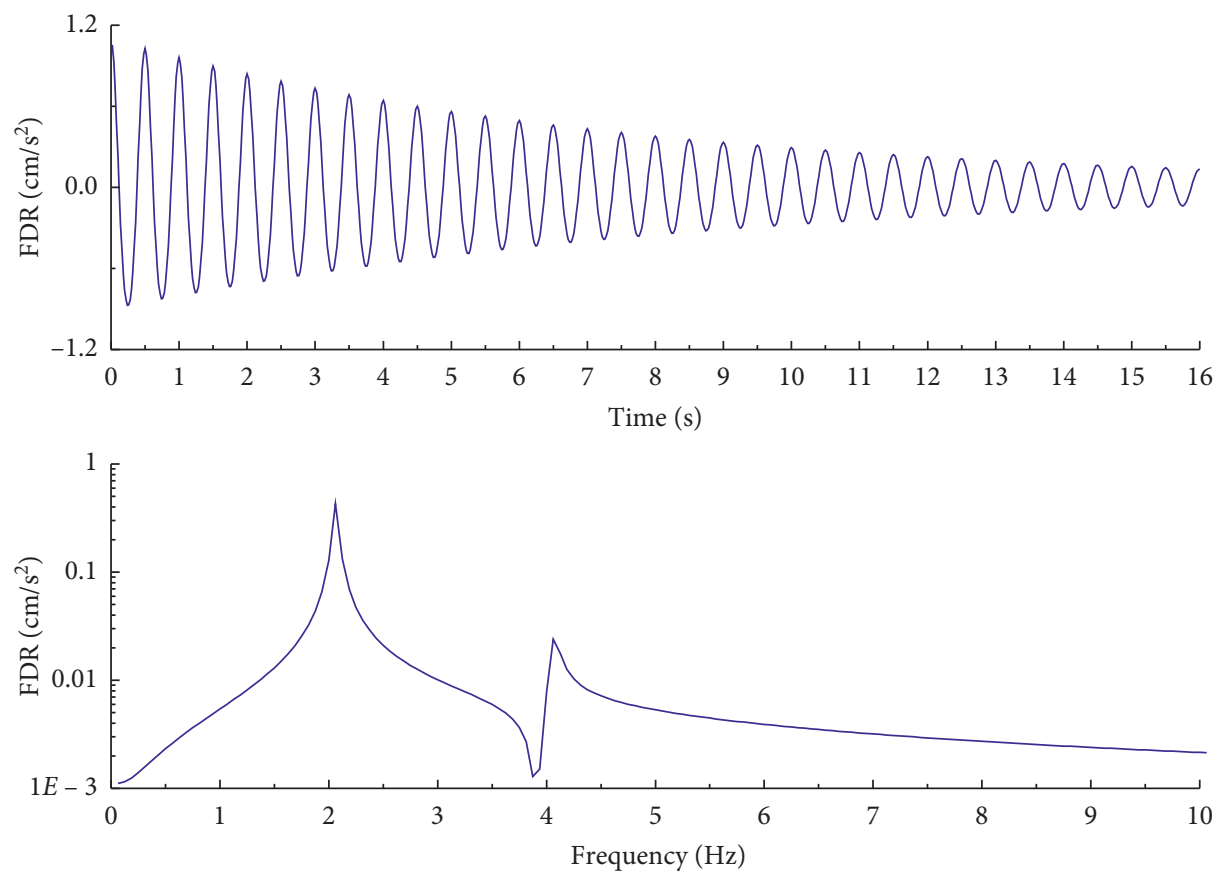

FIgURE 5: Two-component signal with low-energy mode.

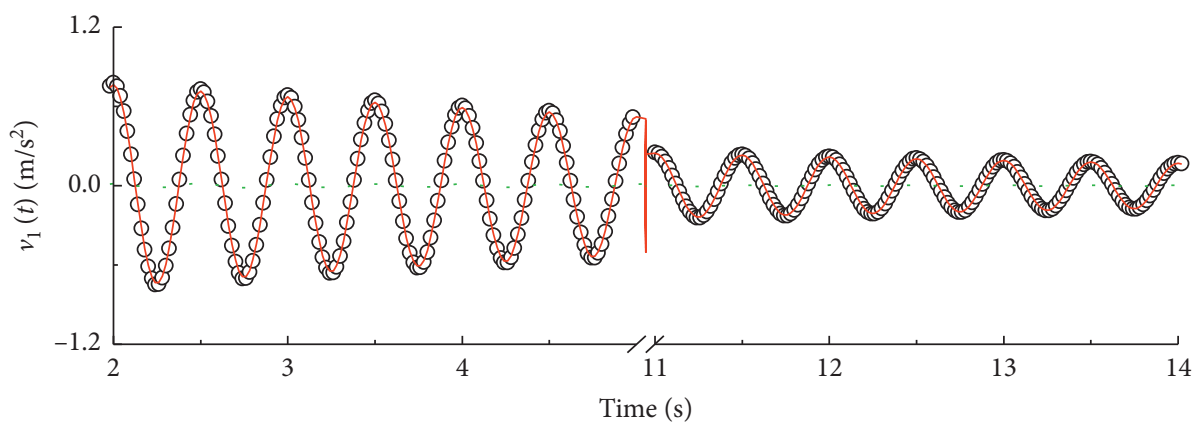

○ True value

- HVD

... Error

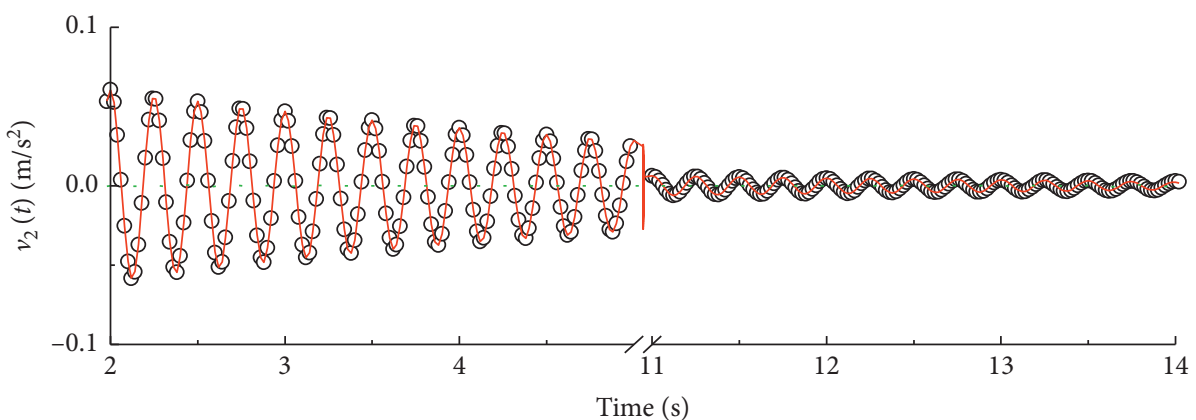

- True value

- HVD

Error

Figure 6: Modal responses of a 2 DOF system (HVD results). 


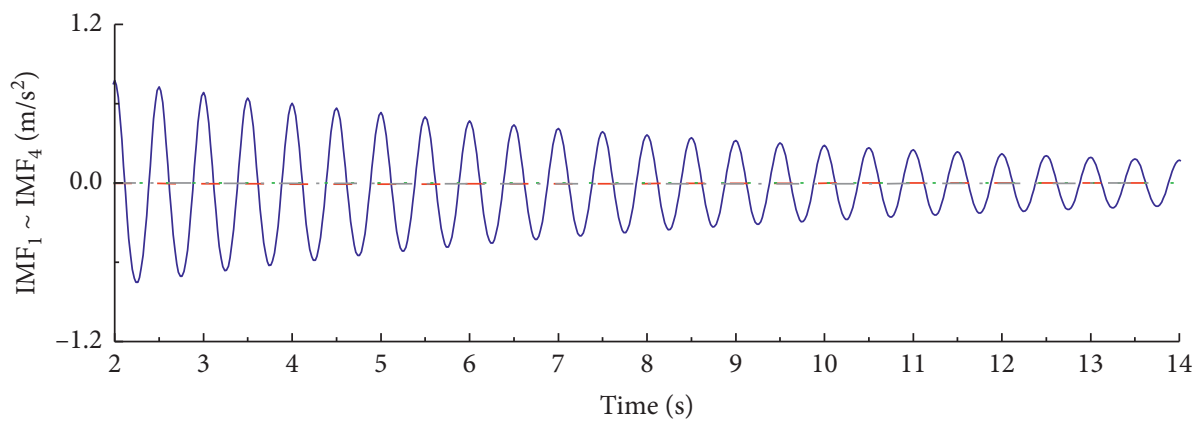
$-\mathrm{IMF}_{1}$
.... $\mathrm{IMF}_{3}$
--- $\mathrm{IMF}_{2}$
-.- $\mathrm{IMF}_{4}$

है

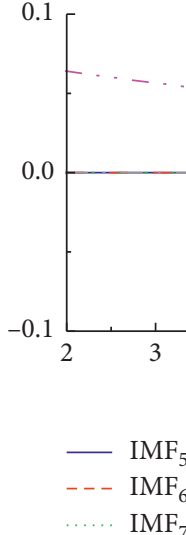

$\mathrm{IMF}_{7}$

Figure 7: EMD results.
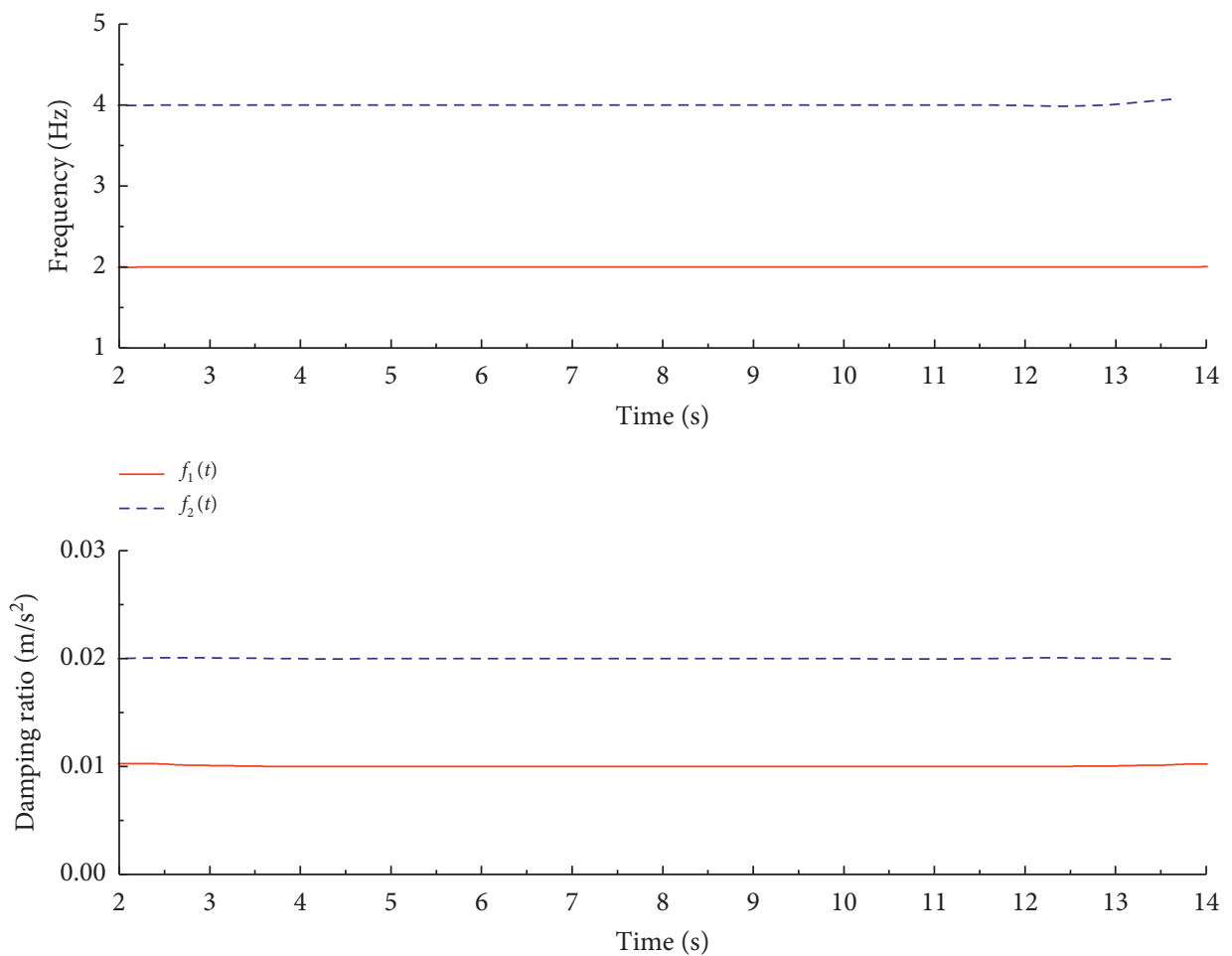

$-\xi_{1}(t)$

- - $\xi_{2}(t)+0.01$

FIGURE 8: Instantaneous frequency and damping ratio with low-energy mode. 
TABLE 3: Identification results for various SNRs with low-energy mode.

\begin{tabular}{lcccc}
\hline SNR $(\mathrm{db})$ & $f_{1}$ & $f_{2}$ & $\xi_{1}$ & $\xi_{2}$ \\
\hline$+\infty$ & 2.00 & 4.00 & 0.010 & 0.010 \\
40 & 2.00 & 4.00 & 0.010 & 0.010 \\
30 & 2.00 & 3.95 & 0.010 & 0.010 \\
20 & 2.00 & 3.91 & 0.010 & 0.011 \\
10 & 2.00 & 3.82 & 0.010 & 0.012 \\
\hline
\end{tabular}

Note. $\xi_{1}$ and $\xi_{2}$ are the modal damping and $f_{1}$ and $f_{2}$ are the modal frequency.

decomposition result of the low-energy modal response is easily disturbed by noise, so its parameter identification is more sensitive to noise.

\section{Conclusions}

A new method for structural modal parameter identification based on the HVD method was proposed. The HVD method overcomes some defects of the EMD method, such as the inability to effectively decompose dense modes and lowenergy modes, so it is more suitable for the decomposition and time-frequency analysis of specific types of nonlinear nonstationary signals. To identify the potential nonlinear characteristics of modal parameters, the instantaneous frequency of the modal response is calculated using the empirical envelope method, and the instantaneous damping ratio is calculated based on the instantaneous logarithmic decay rate of modal response amplitude.

Through a series of numerical examples, the effectiveness of this method was verified, the advantages of this method compared with the EMD-based recognition method were highlighted, and the antinoise performance of this method was studied. The results showed that this method can be used to identify the modal parameters of linear and nonlinear systems, dense modal systems, and low-energy modal systems.

\section{Data Availability}

The calculated data used to support the findings of this study are included within the article.

\section{Conflicts of Interest}

The authors declare no conflicts of interest.

\section{Authors' Contributions}

Yingzhi Xia conceptualized, developed methodology, wrote the original draft, investigated the study, and carried out data curation. Hui Li conceptualized the study, developed methodology, investigated the study, and carried out data curation. Zhezhe Fan investigated the study and carried out data curation. Jiyong Xiao helped with resources.

\section{Acknowledgments}

The authors thank the support of the Science and Technology Project of Henan Province under Grant no. 173102310387.

\section{References}

[1] A. Bagheri, E. O. Osman, and K. H. Devin, "Structural system identification based on variational mode decomposition," Journal of Sound and Vibration, vol. 417, pp. 182-197, 2018.

[2] T. Kijewski, "A Kareem, "Wavelet transforms for system identification in civil engineering," Computer Aided Civil and Infrastructure Engineering, vol. 18, no. 5, pp. 339-355, 2003.

[3] J. N. Yang, Y. Lei, S. Pan et al., "System identification of linear structures based on Hilbert-Huang spectral analysis. Part 1: normal modes," Earthquake Engineering and Structural Dynamics, vol. 32, no. 9, pp. 1443-1467, 2003.

[4] N. E. Huang, Z. Shen, S. R. Long et al., "The empirical mode decomposition and the Hilbert spectrum for nonlinear and non-stationary time series analysis," Proceedings of the Royal Society of London. Series A: Mathematical, Physical and Engineering Sciences, vol. 454, no. 1971, pp. 903-995, 1998.

[5] C. Bao, H. Hong, X. Zhong et al., "Time-varying system identification using a newly improved HHT algorithm," Computers and Structures, vol. 87, no. 23-24, pp. 1611-1623, 2009.

[6] M. Feldman, "Time-varying vibration decomposition and analysis based on the Hilbert transform," Journal of Sound and Vibration, vol. 295, no. 3-5, pp. 518-530, 2006.

[7] M. Feldman, "Theoretical analysis and comparison of the Hilbert transform decomposition methods," Mechanical Systems and Signal Processing, vol. 22, no. 3, pp. 509-519, 2008.

[8] M. Feldman, "Hilbert transform in vibration analysis," Mechanical Systems and Signal Processing, vol. 25, no. 3, pp. 735-802, 2011.

[9] X. Zhu and P. Zhou, "Research on fault diagnosis method of rotor vibration based on kl-hvd," Vibration and Shock, vol. 37, no. 16, pp. 249-255, 2018.

[10] N. Huang, Z. Wu, S. Long et al., "On instantaneous frequency," Advances in Adaptive Data Analysis, vol. 1, no. 2, pp. 177-229, 2009.

[11] J. Zhen and J. Cheng, "A new method of estimating instantaneous frequency empirical envelope method," Vibration and Shock, vol. 31, no. 17, pp. 86-90, 2012.

[12] M. Zhang, "Parameter identification of nonlinear system based on empirical envelope method," Journal of Computational Mechanics, vol. 1, pp. 123-127, 2018.

[13] M. Zhang and F. Xu, "Nonlinear vibration characteristics of bridge deck section models in still air," Journal of Bridge Engineering, vol. 23, no. 9, Article ID 04018059, 2018.

[14] M. Zhang and F. Xu, "Variational mode decomposition based modal parameter identification in civil engineering," Frontiers of Structural and Civil Engineering, vol. 13, no. 5, pp. 10821094, 2019. 